IdeAs

Idées d'Amériques

$10 \mid 2017$

États-Unis / Cuba : une nouvelle donne?

\title{
Causes structurelles de l'enrichissement personnel au Venezuela : relations institutionnelles et les nouveaux riches de la révolution bolivarienne
}

\section{Eduardo Ríos Ludena}

\section{CpenEdition}

Journals

Édition électronique

URL : https://journals.openedition.org/ideas/2215

DOI : 10.4000/ideas.2215

ISSN : 1950-5701

Éditeur

Institut des Amériques

\section{Référence électronique}

Eduardo Ríos Ludena, «Causes structurelles de l'enrichissement personnel au Venezuela : relations institutionnelles et les nouveaux riches de la révolution bolivarienne », IdeAs [En ligne], 10 | 2017, mis en ligne le 19 décembre 2017, consulté le 20 octobre 2022. URL : http://journals.openedition.org/ ideas/2215; DOI : https://doi.org/10.4000/ideas.2215

Ce document a été généré automatiquement le 20 octobre 2022

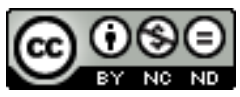

Creative Commons - Attribution - Pas d'Utilisation Commerciale - Pas de Modification 4.0 International - CC BY-NC-ND 4.0

https://creativecommons.org/licenses/by-nc-nd/4.0/ 


\title{
Causes structurelles de
} l'enrichissement personnel au Venezuela : relations institutionnelles et les nouveaux riches de la révolution bolivarienne

\author{
Eduardo Ríos Ludena
}

\section{Introduction}

1 Les preuves d'enrichissement personnel d'hommes d'affaires connectés au gouvernement vénézuélien qui foisonnent dans la presse, ont ressurgi lors de ma recherche de terrain de douze mois à l'occasion de ma thèse doctorale au Venezuela. Des hommes d'affaires me demandaient de couper l'enregistrement pour donner, « off the record ", des détails sur ces fortunes qui explosaient à l'ombre de l'État pétrolier ${ }^{1}$. Des journalistes d'affaires me faisaient part de contrats d'importations d'armes entre les mains de nouveaux riches notoires. Des journalistes des pages sociales rapportaient des fêtes extravagantes de personnes enrichies par la revente de bons de la république lors desquelles les invités d'honneur ressortaient avec des portables américains en remerciement de leur présence dans les salons de fête les plus exclusifs de la capitale. A l'occasion d'une lettre ouverte suite à sa destitution par Nicolás Maduro, le ministre du Plan d'Hugo Chávez, Jorge Giordani, a écrit qu'au moins 300 milliards de dollars auraient été dérobés à l'État vénézuélien par des sociétés écrans d'hommes d'affaires liés au gouvernement (Giordani J., 2014). La dernière de ces manipulations financières a vu une société de courtage inconnue (Dinausaur Group) se porter intermédiaire d'une opération de revente des bons de la république à, la banque d'investissement Goldman Sachs, à 69\% du prix de vente pour 865 millions de dollars ; l'initiateur de l'opération était la Banque centrale du Venezuela. 
2 L'évidence de cette multiplication d'occasions pendant lesquelles des individus liés au gouvernement se sont enrichis personnellement grâce à leurs rapports personnels avec des bureaucrates se proclamant du socialisme du XXI ${ }^{\text {ème }}$ siècle, est souvent reprise par le discours politique. Des articles des deux camps de l'échiquier dénoncent régulièrement ces abcès de corruption. Cette critique - politique ou journalistique - de cas particuliers obscurcit les effets structurels qui les ont générés et qui ont été maintenus par des logiques d'inertie institutionnelle par la révolution bolivarienne. Cet article entreprend de dévoiler les causes structurelles de cet enrichissement personnel. $\mathrm{Au}$ centre de cette nouvelle logique structurelle se situe la réponse d'Hugo Chávez au coup d'Etat de 2002 puis à la grève générale organisée par le patronat fin 2002-début 2003. Suite à ces évènements, Chávez a coupé les ponts avec ce qu'il considérait être le monde d'affaires "traditionnel» dans le pays et a commencé à contrôler plus strictement l'attribution des dollars générés par l'industrie pétrolière à travers le système du contrôle des changes. Cette alchimie institutionnelle a réduit l'échange entre le gouvernement et le secteur privé à une relation avec des compagnies séparées dont l'objectif était l'attribution de pétrodollars. L'exemple des nouveaux riches à particule (les bolibourgeois et les bolichicos) illustre ces effets qui font partie d'un modèle structurel plus large. La relation entre le secteur privé et le gouvernement d'Hugo Chávez demande l'introduction d'une chronologie différente de celle normalement établie par les textes de sciences sociales sur la fin du bipartisme et de la IV ${ }^{\text {ème }}$ république qui le faisait vivre.

\section{La rupture de 2002-2003}

3 Le patronat dit «traditionnel» au Venezuela était particulièrement amoindri lorsqu'Hugo Chávez a pris le pouvoir en 1999. Contre l'idée reçue des processus de libéralisation économique en Amérique latine, l'apertura au Venezuela a réduit le pouvoir des grandes familles économiques de la deuxième moitié du XX⿳亠丷⿵冂⿱十口犬e siècle. La compétition avec les compagnies étrangères et la libéralisation des taux d'intérêts ont érodé leur emprise sur le monde économique. Ce régime de libéralisation, sans agences régulatrices performantes pour en cadrer les conséquences, a enfanté la crise bancaire de 1994, la plus importante en pourcentage d'actifs affectés du XX ${ }^{\text {ème }}$ siècle. Cette crise a non seulement stoppé la croissance économique - au travers de l'interconnexion du monde financier avec d'autres secteurs comme le secteur commercial ou le secteur de la construction, par exemple. Elle a également réduit les faibles ressources dont disposait l'État vénézuélien et a profondément divisé le monde économique de l'époque. Le secteur bancaire le plus affecté a financé Chávez lors de sa campagne de 1998 ; Chávez a reçu des fonds d'un des milliardaires du pays - qui craignait les conséquences potentielles que l'arrivée au pouvoir de son adversaire le plus direct (Henrique Salas Römer) pourrait avoir sur leurs intérêts économiques. Salas Römer, le candidat en deuxième position lors des élections de 1998, était proche des milieux bancaires les moins affectés par la crise bancaire (Gates L., 2010).

Chávez est arrivé au pouvoir en 1999 sans avoir entretenu une relation de long terme avec les hommes d'affaires, mais avec des liens qu'il a tenté d'améliorer lors de ses premières années de gouvernement. Lors d'entretiens avec des journalistes économiques, des fonctionnaires dans les ministères chargés des affaires éconmiques et des hommes d'affaires qui ont eu des rapports avec l'État pendant ces premières 
années, j'ai pu recueillir des rapports concordants qui faisaient part de relations fluides avec le secteur privé entre 1999 et début 2003. Le gouvernement accompagnait les délégations $\mathrm{du}$ syndicat patronal (Fedecámaras) lors de négociations dans les Amériques. Le chavisme au pouvoir a fait voter la loi de libéralisation du marché des télécommunications qui a ouvert le marché (il y avait une entreprise, ancien monopole public, et une compagnie détenue par un des milliardaires du pays) aux investisseurs internationaux. Cette loi était dans la continuation directe de la première loi de télécommunications votée par Carlos Andrés Pérez dans les années 1990. Le début des années 2000 a également vu la consolidation de groupes bancaires dont un qui a permis à Juan Carlos Escotet de devenir, à terme, le troisième milliardaire du pays.

5 Á la suite des décrets-lois de 2001 (leyes habilitantes), le patronat a commencé à se distancer d'Hugo Chávez. Cette confrontation a atteint son acmé lors du coup d'État de 2002 qui a porté le patron de Fedecámaras à la tête du pays pendant quelques heures², puis de la grève générale organisée par les patrons et les managers de l'industrie pétrolière d'Etat. Á la suite de ces confrontations, Chávez a coupé les relations institutionnelles avec le secteur privé «traditionnel » et a instauré un régime de taux de changes géré par Cadivi (Commission d'administration des devises). Le monde économique du début des années 2000 n'était pas un monde dominé par une vieille oligarchie qui se serait fait détrôner par Hugo Chávez. Le chavisme s'est imposé sur un monde économique recomposé, formé en partie par les nouvelles fortunes des années 1980 et 1990. Du point de vue du champ économique, c'est ce nouveau monde qui s'est confronté à Hugo Chávez en 2002-2003 ; c'est ce nouveau monde que Chávez a cherché à apprivoiser.

6 Cadivi a été créée pour faire face à la fuite de capitaux provoquée par l'incertitude politique de l'année 2002. L'agence attribuait des dollars aux hommes d'affaires et aux particuliers qui déposaient un dossier de demande. Cette solution a permis de réduire l'offre dans le marché des changes dans le pays, en même temps qu'elle donnait les moyens au gouvernement de contrôler discrétionnairement l'attribution de dollars aux compagnies. La concomitance d'une rupture des liens institutionnels - ou réglementaires - avec le monde des affaires et de la création d'une agence qui contrôlait l'attribution de dollars au cas par cas a généré une structure économique qui donnait un avantage considérable aux hommes d'affaires qui parvenaient à discuter avec des membres du gouvernement. Cet avantage leur permettait d'accéder à des informations privilégiées mais aussi de revendre leurs dollars sur le marché noir pour gonfler leurs marges. Ces dollars pouvaient être obtenus à travers l'achat de bons de la république, par l'obtention de contrats en monnaie étrangère et aussi par l'obtention de dollars qui étaient écoulés via des compagnies écran dont le seul but était l'accès aux fonds publics.

7 L'apparition de la bolibourgeoisie - la bourgeoisie de la révolution bolivarienne - est la preuve la plus évidente de ce système qui récompensait l'accès direct aux ministères par l'enrichissement personnel; système qui a été maintenu sous Nicolás Maduro. Cependant, ces cas médiatisés ne permettent pas de saisir comment ces inter-actions ont contribué à la création d'une structure économique qui entretenait la séparation entre les hommes d'affaires liés au gouvernement et le reste du monde économique créant de fait des intérêts puissants qui ont empêché toute transformation du monde économique au moment de la crise à partir de 2012. 


\section{Une fenêtre d'opportunité politique : l'apparition des bolibourgeois}

8 La bolibourgeoisie permet de donner un exemple concret des logiques structurales que nous décrirons ci-dessous. Le nom bolibourgeoisie a été donné par un journaliste économique en référence aux bénéfices directs qu'ont retirés une série d'hommes d'affaires suite à la rupture entre Chávez et le patronat dit «traditionnel». Les exemples de Ricardo Fernandez Barruecos et Willmer Ruperti mettent en évidence comment des «fenêtres d'opportunités politiques» ont donné pour résultat l'accumulation personnelle de biens économiques.

9 L'histoire de leur entrée dans le monde des affaires précède l'ascension d'Hugo Chávez. Cette idée de nouveaux riches est un déguisement utile qui sert leurs détracteurs dans le monde des affaires - qui peuvent se poser en représentants du vrai secteur privé - et un discours politique de gauche révolutionnaire qui peut ainsi prétendre que le gouvernement Chávez n'a jamais entretenu de relations avec des hommes d'affaires installés de longue date dans le pays. Willmer Ruperti avait intégré l'école de marine marchande et s'est spécialisé dans le transport pétrolier dès les annés 1990. Fernandez Barruecos avait une présence dans le monde des affaires à travers des investissements dans l'industrie thonière et les parkings. Les deux avaient également des contacts avec le gouvernement dès avant l'arrivée de Chávez. Fernandez Barruecos avait aidé à la mise en place de supermarchés subventionnés par l'État (supermercados casa) lors du gouvernement de Rafael Caldera (1994-98). Willmer Ruperti finançait des « hommes de l'ombre» de l'aile gauche du gouvernement Caldera. Bien que faisant partie de ce milieu d'affaires, leur fortune a explosé sous Hugo Chávez.

En 2002-2003, lors de la grève générale qui a provoqué une chute du PIB de 19\% sur deux ans, le gouvernement a dû faire face à deux problèmes majeurs. Premièrement, la question pratique d'alimenter et de fournir le pays en pétrole. Deuxièmement, créer des lignes d'approvisionnement en nourriture qui étaient normalement servies par le secteur privé en grève. Fernandez Barruecos, qui avait déjà aidé le père d'Hugo Chávez (gouverneur de l'État de Barinas) à résoudre une crise alimentaire en 2000, a été appelé pour établir un plan de ravitaillement en urgence. Willmer Ruperti a rétabli les importations de pétrole dans le pays. Les deux ont fait un pari politique considérable en soutenant Hugo Chávez. Fernandez Barruecos a aidé le gouvernement à racheter les machines invendues auprès des compagnies productrices de camions de charge et à acheter des stocks dans les pays voisin pour fournir le pays. D'ailleurs, il s'est associé avec le narcotrafiquant Walid Mackled pour monter cette ligne de distribution. Willmer Ruperti a déplacé les navires qui empêchaient l'accès aux ports pétroliers vénézuéliens, et a même payé lui-même les premiers tankers qui ont fourni le Venezuela. En partie grâce à leur aide, le gouvernement a survécu à l'épreuve politique.

11 Les services rendus par Willmer Ruperti lui ont permis d'accéder au contrat de transport de pétrole de PDVSA vers les États-Unis pendant dix ans. Contrat qu'il a titrisé et revendu à la bourse de Caracas. Il a récupéré ses investissements dans les six mois. Il a notamment acheté une chaîne de télévision pour empêcher que l'opposition n'ait accès aux ondes de diffusion dans le pays. Fernandez Barruecos a obtenu le contrat de farine de maïs (produit de base de l'alimentation vénézuélienne) pour suppléer la mission Mercal qui, à son apogée, alimentait quotidiennement 13 millions de Vénézuéliens. On estime que les fortunes de Willmer Ruperti et Ricardo Fernández 
Barruecos ont atteint le milliard de dollars sous Hugo Chávez. Ricardo Fernandez Barruecos a été poursuivi en 2009 par ce dernier après s'être diversifié dans le monde bancaire. Willmer Ruperti continue à opérer dans le pays.

12 Le même type de circonstances exceptionnelles a permis à la compagnie Derwick de gagner ses premiers contrats pour la construction d'usines électriques dans le pays. Alors que le gouvernement faisait face à une crise énergétique sévère et déboursait 40 milliards de dollars sur deux ans, ces bolichicos, ou bolibourgeois de deuxième génération, se sont enrichis massivement et très rapidement. Ces très jeunes hommes d'affaires aident à faire vivre le mythe de cette nouvelle bourgeoisie enrichie par Chávez. Ils étaient tous de jeunes hommes sortis des meilleures universités du pays avec de longues histoires familiales dans le monde intellectuel vénézuélien. Ce que ces cas particuliers mettent en évidence est bien plus la solution politique trouvée par Hugo Chávez pour s'accommoder du monde économique existant que la preuve du bouleversement total qu'il aurait provoqué.

13 Ces exemples d'hommes d'affaires - il y avait très peu de femmes - qui ont profité de ces fenêtres d'opportunité politiques permettent de comprendre les raisons structurelles qui ont permis leur éclosion. Les exemples ponctuels d'enrichissement massif peuvent être interprétés comme des exceptions en temps de crise. Ils le sont par les responsables politiques proches du gouvernement cherchant à en limiter la portée. Cependant, pour paraphraser Michel Dobry, les crises mettent en évidence les caractéristiques profondes d'un système.

\section{L'entretien de logiques structurelles d'enrichissement personnel}

14 Le système d'enrichissement personnel permis par la reconfiguration post-2002 a généré une structure structurante, en ce qu'elle avait un effet d'inertie. Elle formait un Conatus au sens de Spinoza. L'exemple de la transformation de Fedecámaras (la fédération nationale des chambres de commerce et d'industrie dans le pays) après le coup d'État et la grève générale permet de passer d'exemples de corruption individuelle à une analyse préliminaire des structures économiques qui les ont produites. Le passage par une analyse en termes de carrières permet de montrer que les positions de pouvoir occupées par un agent dans le monde économique sont le produit de longues carrières qui agissent comme un processus de sélection à l'intersection de filtres opérés par le système scolaire, par les structures d'Etat et par les grandes organisations économiques elles-mêmes, avec un poids différent de chacun de ces facteurs selon le cas individuel observé. Si ces résultats sont classiques dans l'analyse du monde économique européen et nord-américain, elles sont encore parcellaires en ce qui concerne l'analyse des terrains latino-américains.

15 Pour accéder à la présidence de Fedecámaras il faut avoir occupé tous les rôles hiérarchiques dans les organisations de base, puis dans les organisations sectorielles, puis les postes de direction de la fédération elle-même. Vu que Fedecámaras est une organisation pro bono, les présidents de Fedecámaras ont non seulement vingt ans de carrière "syndicale ", ils ont également vingt ans de pratique du monde des affaires vénézuélien. Ce processus de spécialisation dévoile un monde économique au Venezuela qui n'est pas uniforme, sans pour autant être rigide. Ces viscosités 
permettent d'expliquer d'une part la transformation qui s'est opérée au sein du monde économique privé au Venezuela et d'autre part en quoi cette transformation a contribué d'autant plus à solidifier la structure économique.

Exclus d'un accès institutionnel à l'État, les hommes d'affaires non-alignés avec le gouvernement se sont tournés vers une pratique économique qui permettait de capturer la rente pétrolière indirectement. Alors que les programmes sociaux de Chávez ont permis l'augmentation du pouvoir d'achat des plus démunis et des classes moyennes, les hommes d'affaires qui ne recevaient pas systématiquement de dollars de Cadivi ont restructuré leurs affaires pour écouler leurs marchandises à ces nouvelles classes moyennes et populaires financées par les prix du pétrole. Cette transformation a été mise en évidence dans l'accès à la présidence de Fedecámaras d'un nouveau groupe d'hommes d'affaires qui avaient construit leurs carrières pendant la libéralisation économique des années 1990 et qui avait été exclus de l'accès direct au fonds de l'État depuis l'arrivée de Chávez au pouvoir. Un de ces hommes d'affaires, pour l'anecdote, avait été président de la faction parlementaire d'un des partis de l'extrêmegauche pendant la quatrième république, La Cause radicale.

Cette analyse idéale-typique qui oppose, d'une part, des hommes d'affaires proches des structures de représentation du patronat qui accèdent indirectement à la rente pétrolière à, d'autre part, des hommes d'affaires connectés entreprise par entreprise au gouvernement et qui profitent de leurs contacts pour accéder à des dollars subventionnés, est réductrice, comme toute analyse idéale-typique ${ }^{3}$. Elle néglige par exemple l'importance de la relation très spéciale que le gouvernement entretenait avec les compagnies multinationales, pétrolières et non-pétrolières. Elle permet nonobstant de montrer les effets d'inertie de cette structure économique en temps de crise sous Nicolás Maduro.

18 La bolibourgeoisie est un groupe d'hommes d'affaires dont l'avantage comparatif - non pas la logique fonctionnelle - est l'accès aux fonds de l'Etat. L'autre groupe, dont l'avantage comparatif est de s'adapter au monde économique vénézuélien " indirectement", a pu utiliser bien plus facilement cette spécialisation lorsque la situation économique s'est dégradée et que les comptes publics se sont réduits considérablement. Ces hommes d'affaires liés au gouvernement se sont retrouvés dans une confrontation économique avec des hommes d'affaires bien mieux équipés qu'eux, pour ceux qui ont réussi à survivre aux années Chávez ${ }^{4}$. Ce désavantage pouvait également être préjudiciel pour le gouvernement qui risquait ainsi de perdre très rapidement ses réseaux d'hommes d'affaires qu'il avait construits avec difficulté. En temps de crise économique, ces hommes d'affaires liés au gouvernement sont ainsi devenus des vecteurs de soutien à cette structure économique qui leur promet encore l'enrichissement personnel. C'est pourquoi, après $30 \%$ de chute du PIB sur quatre ans de gouvernement Maduro et avec une augmentation de la pauvreté dramatique, des cas de corruption continuent à fuiter dans la presse. C'est l'évidence que le gouvernement a mis en place une structure structurante du monde économique vénézuélien qui garde encore de son inertie - par un pur effet d'hystérésis -, alors que les conditions économiques la rendent contreproductive. 


\section{Conclusion : fragments pour une sociologie de la corruption}

19 Tout système normatif circonscrit les modalités de violation de sa propre norme. Les excès repérés dans les médias et par la critique politique ne sont que des mises en évidence des effets normatifs qui soutiennent la structure qui produit la violation de la norme. La modalité de violation de la norme d'un système économique qui récompense l'accès individuel par l'enrichissement personnel est souvent qualifié de corruption. En cela, Chávez n'a pas inventé la corruption dans le pays. La quatrième république s'est délégitimée par accusations de corruptions interposées. Il a simplement remis en place un système d'incitations légales qui favorisait la corruption dans un monde économique qui avait une longue expérience dans la gestion des fonds publics. Toute chronique de la relation entre le secteur privé et l'État sous Hugo Chávez doit se lire dans cette rencontre entre les incitations d'Etat et les spécialisations du monde économique vénézuélien. Les bolibourgeois ne sont peut-être qu'anecdotiques, mais ils sont la porte d'entrée à une structure économique complexe que la chronique journalistique se refuse de voir en se concentrant sur des cas de corruption individuelle.

\section{BIBLIOGRAPHIE}

Coronil, Fernando, «State Reflections: The 2002 Coup against Hugo Chávez », in Thomas Ponniah et Jonathan Eastwood (dir.), The Revolution in Venezuela: Social and Political Change Under Chávez, Cambridge, MA, Harvard University David Rockefeller Center for Latin American Studies, 2011, p. 37-65.

Gates, Leslie, Electing Chavez: The Business of Anti-neoliberal Politics in Venezuela, Pittsburgh, University of Pittsburgh Press, 2010.

Giordani, Jorge, « Testimonio y responsabiliad ante la historia », aporrea, 18/06/2014. https:// www.aporrea.org/ideologia/a190011.html, page consultée le 7 décembre 2017.

Rios Ludena, Eduardo, Embedding industrial policy in oil-states : The mixed-effects of an uncoordinated state and the strategies of the Venezuelan business elites at the close of an oil boom (2012-2014), Thèse de doctorat, Sciences Po Paris, 2016.

\section{NOTES}

1. Dans ce qui suit, les informations ont été données par les plus de 60 entretiens menés au Venezuela auprès de sources proches du monde des affaires dans le pays.

2. Il est possible de discuter les raisons pour lesquelles Pedro Carmona Estanga est arrivé à la présidence de la république. Il ne semble cependant pas être le «cerveau » du coup d'Etat (Coronil F., 2011). 
3. Pour une analyse bien plus longue des oppositions dans le monde économique vénézuélien veuillez vous référer à ma thèse de doctorat (Rios Ludena E., 2016).

4. Comme dans tout processus économique, de nombreux hommes d'affaires ont été très fortement attaqués par le gouvernement et ont soit fermé leurs entreprises soit vu leurs compagnies se faire exproprier par le gouvernement.

\section{AUTEUR}

\section{EDUARDO RÍOS LUDENA}

Eduardo Rios est docteur en sciences Politiques de Sciences Po Paris/Ceri (CNRS) où il a écrit sa these de doctorat sur les élites économiques et les politiques industrielles des états pétroliers. Il se prepare à publier des travaux sur les strategies des hommes d'affaires dans les états pétroliers et la structure du monde économique au Venezuela sous Hugo Chávez. 